Part of Journal of Research of the National Bureau of Standards, Volume 33, November 1944

\title{
THERMAL-DENSITY COEFFICIENTS AND HYDROMETER CORRECTION TABLES FOR VEGETABLE TANNING EXTRACTS
}

\author{
By Mary Grace Blair and Elmer L. Peffer
}

\begin{abstract}
Densities and thermal expansions have been determined of the vegetable tanning extracts-quebracho, oak bark, hemlock bark, chestnut, and mangrove bark - within the range 1.00 to 1.12 specific gravity at $60^{\circ} / 60^{\circ} \mathrm{F}$ and over the temperature range $50^{\circ}$ to $100^{\circ} \mathrm{F}$. Thermal-density coefficients are presented in such a manner that the density of an extract at any temperature within the range can be calculated if its specific gravity at $60^{\circ} / 60^{\circ} \mathrm{F}$ or its density at $25^{\circ} \mathrm{C}$ is known. The information obtained has been used also in the preparation of tables for correcting hydrometer readings at observed temperatures in degrees barkometer, in degrees Twaddle, and in degrees Baumé to readings at the standard temperature $60^{\circ} \mathrm{F}$.
\end{abstract}

\section{CONTENTS}

Page

I. Introduction

341

II. Procedure

III. Reduction of observations _._.

IV. Transformation of data to $60^{\circ} \mathrm{F}$

V. Calculation of hydrometer corrections _.

VI. Applicability and accuracy of the coefficients and derived correction tables....

VII. Conclusion

\section{INTRODUC'TION}

In the leather-tanning industry the specific gravity of a tanning extract is used in conjunction with other data accumulated for that particular extract to measure the strength of the tannins present. Three types of hydrometers with scales based on specific gravitybarkometer, Twaddle (also spelled Twaddell), and Baumé-are in common industrial use. Each of these hydrometers is standard at $60^{\circ} \mathrm{F}$. In general, it is impracticable to cool each sample to this temperature before observations are made. The much quicker method of reading the hydrometer at any temperature and applying a correction to obtain the true value at the standard temperature is preferred.

In view of the extensive use of hydrometers at tanneries, The American Leather Chemists Association suggested that the National Bureau of Standards check the barkometer correction table in most common use, extend this table, and prepare tables for Twaddle and Baumé hydrometers. The Association supplied samples of extracts considered to be representative of the chief tanning extracts. The samples 
included hemlock bark, oak bark, quebracho, chestnut, and mangrove bark ("cutch") extracts. Thermal-density coefficients of these extracts were determined and are presented in such a manner that they may be used to change true specific gravity at $60^{\circ} / 60^{\circ} \mathrm{F}$ to density or specific gravity at other temperatures. Their application in the calculation of hydrometer corrections is also discussed. A table for use in correcting the readings of hydrometers graduated in specific gravity units is presented. The values for barkometer, Twaddle, and Baumé scales calculated from these results will be found in tabular form in National Bureau of Standards Circular C449, Hydrometer Correction Tables and Thermal Density Coefficients for Vegetable Tanning Extracts.

\section{PROCEDURE}

As the tanning extracts are used in various strengths, density determinations were made of diluted samples covering the range of the barkometer hydrometer, 0 to 120 degrees barkometer or 1.00 to 1.12 specific gravity at $60^{\circ} / 60^{\circ} \mathrm{F}$. Water in sufficient amounts, calculated with the original density of the extracts as the basis, was added to known weights of the extracts to produce samples with specific gravities of approximately 1.03, 1.06, 1.09, and 1.12. One sample, mangrove bark extract, which was supplied in solid form, was dissolved and diluted to the required specific gravity by the aid of the hydrometer.

Diluting the samples caused precipitation of colloidal matter in most cases, the amount of precipitation increasing with the extent of the dilution. The picnometers were filled while these finely divided solids were still homogeneously mixed with the solution. Their settling to the bottom in subsequent determinations did not produce any apparent irregularities in the data.

Densities were determined at $10^{\circ}, 20^{\circ}, 25^{\circ}, 30^{\circ}$, and $40^{\circ} \mathrm{C}$ by the picnometer method, that is, by weighing a picnometer of known volume filled in a constant temperature bath adjusted to the desired temperature. Calculation of density was made with correction for the buoyancy of air. ${ }^{1}$

\section{REDUCTION OF OBSERVATIONS}

The rate of change of density with change of temperature was calculated from the observed densities by the application of the method of least squares.

It is assumed that the expansion of any sample may be represented by an equation having the form

in which

$$
D_{t}=D_{T}+\alpha_{T}(t-T)+\beta_{T}(t-T)^{2}+\gamma_{T}(t-T)^{3},
$$

$$
\begin{aligned}
D_{t} & =\text { density at any temperature, } t \\
D_{T} & =\text { density at the standard temperature, } T \\
\alpha_{T}, \beta_{T}, \gamma_{T} & =\text { constant coefficients determined for each } \\
& \text { sample. }
\end{aligned}
$$

\footnotetext{
1 More detailed description of the method and the picnometers used may be found in Tech. Pap. BS 77, 8 (1916) and in Bul. BS 9, 371 (1913) S197.
} 
If the observation equations derived by substitution of the observed values in equation 1 are added, an expression is found for $D_{T}$ as a function of the mean density $\left[D_{t}\right]_{m}$. Calculation of the constants of the density equation has been simplified by the symmetrical selection of the temperatures of observation. Thus, the relationship of $D_{T}$ to $\left[D_{t}\right]_{m}$ becomes

$$
D_{T}=\left[D_{t}\right]_{m}-\left[C_{1}^{2}\right]_{m} \beta_{T} .
$$

The observation equations may now be written in the form

$$
C_{1} \alpha_{T}+C_{2} \beta_{T}+C_{3} \gamma_{T}=N .
$$

$\alpha_{T}, \beta_{T}$, and $\gamma_{T}$ are calculated from the normal equations

in which

$$
\begin{aligned}
& \sum C_{1}^{2} \alpha_{T}+\sum C_{1} C_{2} \beta_{T}+\sum C_{1} C_{3} C_{3}=\sum C_{1} N \\
& \sum C_{1} C_{2} \alpha_{T}+\sum C_{2}^{2} \beta_{T}+\sum_{1} C_{2} C_{3} \gamma_{T}=\sum C_{2} N \\
& \sum C_{1} C_{3} \alpha_{T}+\sum C_{2} C_{3} \beta_{T}+\sum C_{3}^{2} \gamma_{T}=\sum C_{3} N,
\end{aligned}
$$

$$
\begin{aligned}
& C_{1}=t-t_{m} \quad\left(t_{m}=\text { mean temperature }\right) \\
& C_{2}=C_{1}^{2}-\left[C_{1}^{2}\right]_{m} \quad\left(\left[C_{1}^{2}\right]_{m}=\text { mean } C_{1}^{2}\right) \\
& C_{3}=C_{1}^{3}{ }^{3} \\
& N=D_{t}-\left[D_{t}\right]_{m} \quad\left(\left[D_{t}\right]_{m}=\text { mean } D_{t}\right) .
\end{aligned}
$$

The various steps in obtaining the constants for expressing density at any temperature in terms of density at $25^{\circ} \mathrm{C}$ are illustrated by the sample calculation, which shows the operation for liquid quebracho extract diluted to approximately $1.06 \mathrm{~g} / \mathrm{ml}$. Table 1 shows computation of the coefficients to be substituted in the normal equations. The normal equations become

$$
\begin{gathered}
500 \alpha_{25}+102500 \gamma_{25}=-0.16945 \\
52500 \beta_{25}=-0.20325 \\
102500 \alpha_{25}+22812500 \gamma_{25}=-34.69225 \\
D_{25}=1.06813-100 \beta_{25} .
\end{gathered}
$$

By solving these equations, the following values of

$\alpha_{25}, \beta_{25}, \gamma_{25}$, and $D_{25}$ are obtained:

$$
\begin{aligned}
& \alpha_{25}=-0.0003440 \\
& \beta_{25}=-0.00000387 \\
& \gamma_{25}=+0.000000025 \\
& D_{25}=1.06852 .
\end{aligned}
$$

These values when substituted in the equation

$$
D_{t}=D_{25}+\alpha_{25}(t-25)+\beta_{25}(t-25)^{2}+\gamma_{25}(t-25)^{3},
$$

which is derived from the general equation 1, give the density values shown in table 2. 
TABLE 1.-Sample of reduction of observations

\begin{tabular}{|c|c|c|c|c|c|c|c|c|c|c|c|c|c|c|}
\hline$t$ & $C_{1}$ & $C_{1^{2}}$ & $C_{2}$ & $C_{3}$ & $C_{1} C_{2}$ & $C_{1} C_{3}$ & $C_{2}{ }^{2}$ & $C_{2} C_{3}$ & $C_{3}{ }^{2}$ & $D$ & $N$ & $C_{1} N$ & $\mathrm{C}_{2} \mathrm{~N}$ & $\mathrm{C}_{3} \mathrm{~N}$ \\
\hline $\begin{array}{l}10 \\
20 \\
25 \\
30 \\
40\end{array}$ & $\begin{array}{r}-15 \\
-5 \\
0 \\
+5 \\
+15\end{array}$ & $\begin{array}{r}225 \\
25 \\
0 \\
25 \\
225 \\
5) 500 \\
100\end{array}$ & $\begin{array}{r}+125 \\
-75 \\
-100 \\
-75 \\
+125\end{array}$ & $\begin{array}{r}-3375 \\
-125 \\
0 \\
+125 \\
+3375\end{array}$ & $\begin{array}{r}-1875 \\
+375 \\
0 \\
-375 \\
+1875 \\
0\end{array}$ & $\begin{array}{r}+50625 \\
+625 \\
0 \\
+625 \\
+50625 \\
102500\end{array}$ & $\begin{array}{r}15625 \\
5625 \\
10000 \\
5625 \\
15625 \\
52500\end{array}$ & $\begin{array}{r}-421875 \\
+9375 \\
0 \\
-9375 \\
+421875 \\
0\end{array}$ & $\begin{array}{r}+11390625 \\
+15625 \\
0 \\
+15625 \\
+11390625 \\
22812500\end{array}$ & $\begin{array}{r}1.07272 \\
1.07013 \\
1.06852 \\
1.06670 \\
1.06257 \\
5) 5.34064 \\
1.06813\end{array}$ & $\begin{array}{r}+0.00459 \\
+.00200 \\
+.00039 \\
-.00143 \\
-.00556\end{array}$ & $\begin{array}{r}-0.06888 \\
-.01002 \\
.00000 \\
-.00715 \\
-.08340 \\
-0.16945\end{array}$ & $\begin{array}{r}+0.57400 \\
-.15030 \\
-.03920 \\
+.10725 \\
-.69500 \\
-0.20325\end{array}$ & $\begin{array}{r}-15.49800 \\
-.25050 \\
.00000 \\
-.17875 \\
-18.76500 \\
-34.69225\end{array}$ \\
\hline
\end{tabular}

TABLE 2.-Calculation of $D_{t}$

\begin{tabular}{|c|c|c|c|c|c|c|c|c|c|}
\hline$t$ & $t-25$ & $(t-25)^{2}$ & $(t-25)^{3}$ & $\alpha(t-25)$ & $\beta(t-25)^{2}$ & $\gamma(t-25)^{3}$ & $\begin{array}{c}D_{t} \\
\text { calculated }\end{array}$ & $\begin{array}{c}D_{t}, \\
\text { observed }\end{array}$ & $\begin{array}{l}\text { Difference } \\
\text { (cal.-obs.) }\end{array}$ \\
\hline $\begin{array}{l}10 \\
20 \\
25 \\
30 \\
40\end{array}$ & $\begin{array}{r}-15 \\
-5 \\
0 \\
+5 \\
+15\end{array}$ & $\begin{array}{r}225 \\
25 \\
0 \\
25 \\
225\end{array}$ & $\begin{array}{r}-3375 \\
-125 \\
0 \\
+125 \\
+3375\end{array}$ & $\begin{array}{r}+0.00516 \\
+.00172 \\
.00000 \\
-.00172 \\
-.00516\end{array}$ & $\begin{array}{r}-0.00087 \\
-.00010 \\
.00000 \\
-.00010 \\
-.00087\end{array}$ & $\begin{array}{r}-0.00009 \\
.00000 \\
.00000 \\
.00000 \\
+.00008\end{array}$ & $\begin{array}{l}1.07272 \\
1.07014 \\
1.06852 \\
1.06670 \\
1.06257\end{array}$ & $\begin{array}{l}1.07272 \\
1.07013 \\
1.06852 \\
1.06670 \\
1.06257\end{array}$ & $\begin{array}{l}0.00000 \\
.00001 \\
.00000 \\
.00000 \\
.00000\end{array}$ \\
\hline
\end{tabular}


TABLE 3.-Thermal-density coefficients for tanning eatracts

\begin{tabular}{|c|c|c|c|c|}
\hline Tanning Extract & $D_{25}$ & $\alpha_{25} \times 10^{8}$ & $\beta_{25} \times 10^{7}$ & $\gamma_{25} \times 10^{3}$ \\
\hline $\begin{array}{l}\text { Quebracho (from International Products Corporation) } \\
\text { Do } \\
\text { Do } \\
\text { Do }\end{array}$ & $\begin{array}{l}1.19642 \\
1.12156 \\
1.09205 \\
1.06111 \\
1.03080\end{array}$ & $\begin{array}{l}-487 \\
-405 \\
-371 \\
-336 \\
-298\end{array}$ & $\begin{array}{l}-28 \\
-34 \\
-35 \\
-41 \\
-43\end{array}$ & $\begin{array}{l}+2 \\
+2 \\
+1 \\
+4 \\
+2\end{array}$ \\
\hline $\begin{array}{l}\text { Quebracho (SM brand) } \\
\text { Do } \\
\text { Do Do } \\
\text { Do }\end{array}$ & $\begin{array}{l}\text { 1. } 22126 \\
\text { 1. } 12170 \\
1.09110 \\
\text { 1. } 06852 \\
\text { 1. } 03101\end{array}$ & $\begin{array}{l}-515 \\
-407 \\
-370 \\
-344 \\
-300\end{array}$ & $\begin{array}{l}-23 \\
-34 \\
-36 \\
-39 \\
-43\end{array}$ & $\begin{array}{l}+1 \\
+2 \\
+1 \\
+2 \\
+3\end{array}$ \\
\hline $\begin{array}{l}\text { Oak bark (from Teas Extract Co.) } \\
\text { Do } \\
\text { Do } \\
\text { Do } \\
\text { Do }\end{array}$ & $\begin{array}{l}\text { 1. } 23230 \\
1.10959 \\
1.08402 \\
1.06100 \\
1.03143\end{array}$ & $\begin{array}{l}-507 \\
-378 \\
-349 \\
-321 \\
-292\end{array}$ & $\begin{array}{l}-33 \\
-37 \\
-40 \\
-42 \\
-47\end{array}$ & $\begin{array}{l}+6 \\
+2 \\
+3 \\
+2 \\
+3\end{array}$ \\
\hline $\begin{array}{l}\text { Hemlock bark (from Teas Extract Co.) } \\
\text { Do } \\
\text { Do } \\
\text { Do } \\
\text { Do }\end{array}$ & $\begin{array}{l}1.18890 \\
1.11899 \\
1.08573 \\
1.06155 \\
1.03067\end{array}$ & $\begin{array}{l}-466 \\
-389 \\
-356 \\
-323 \\
-291\end{array}$ & $\begin{array}{l}-28 \\
-36 \\
-40 \\
-42 \\
-48\end{array}$ & $\begin{array}{l}+2 \\
+5 \\
+5 \\
+2 \\
+4\end{array}$ \\
\hline $\begin{array}{l}\text { Chestnut (from Mead Corporation) } \\
\text { Do } \\
\text { Do } \\
\text { Do }\end{array}$ & $\begin{array}{l}1.11351 \\
1.08500 \\
1.07203 \\
1.03004\end{array}$ & $\begin{array}{l}-384 \\
-351 \\
-334 \\
-296\end{array}$ & $\begin{array}{l}-37 \\
-40 \\
-43 \\
-45\end{array}$ & $\begin{array}{l}+1 \\
+2 \\
+1 \\
+3\end{array}$ \\
\hline $\begin{array}{l}\text { Solid Mangrove bark, "cutch"' } \\
\text { Do } \\
\text { Doon } \\
\text { Do }\end{array}$ & $\begin{array}{l}\text { 1. } 11851 \\
1.08104 \\
\text { 1. } 04903 \\
1.02422\end{array}$ & $\begin{array}{l}-385 \\
-349 \\
-308 \\
-288\end{array}$ & $\begin{array}{l}-33 \\
-39 \\
-43 \\
-45\end{array}$ & $\begin{array}{r}0 \\
+4 \\
+1 \\
+3 \\
+3\end{array}$ \\
\hline
\end{tabular}

The thermal-density coefficients for each sample calculated according to the above method are listed in table 3 and are shown graphically in figure 1 . It will be noted that the $\alpha$ values for the various extracts are close together. The best straight line fitting all the data was determined by the method of least squares. This line is shown in figure 1. Equations of the $\beta$ and $\gamma$ lines of figure 1 were calculated likewise. The lines have the following equations:

$$
\begin{aligned}
\alpha_{25}= & 0.0008586-0.0011184 D_{25}, \\
\beta_{25}= & -0.00001421+0.00000950 D_{25}, \\
\gamma_{25}=+0.0000000297-0.0000000044 D_{25}(=+0.000000025 & \quad+0 \text { er the density range of this project }) .^{2}
\end{aligned}
$$

$$
\begin{gathered}
\text { Calculated from equations } 3 \\
\alpha_{25}=-257 \times 10^{-6} \\
\beta_{25}=-47 \times 10^{-7} \\
\gamma_{25}=+3 \times 10^{-8}
\end{gathered}
$$$$
\text { Calculated from Chappuis' data }
$$$$
\begin{aligned}
& \alpha_{25}=-257 \times 10^{-6} \\
& \beta_{25}=-48 \times 10^{-7} \\
& \gamma_{25}=+3 \times 10^{-8}
\end{aligned}
$$

The equations provide a ready means for obtaining the coefficients $\alpha_{25}, \beta_{25}$, and $\gamma_{25}$ for tanning extracts of any given density within the range of observations. Thus, if the density of a tanning extract is known at $25^{\circ} \mathrm{C}$, its density at any other temperature may be computed.

\footnotetext{
2 The coefficients for water were not included in the derivation of this set of equations. However, Chappuis' data on water recalculated in the same manner, Bul. BS 9, 401 (1913) S197, agree well with those calculated for $D_{25}=0.99708$. The values are not strictly comparable, since Chappuis' values are for air-free water.
} 
346 Journal of Research of the National Bureau of Standards

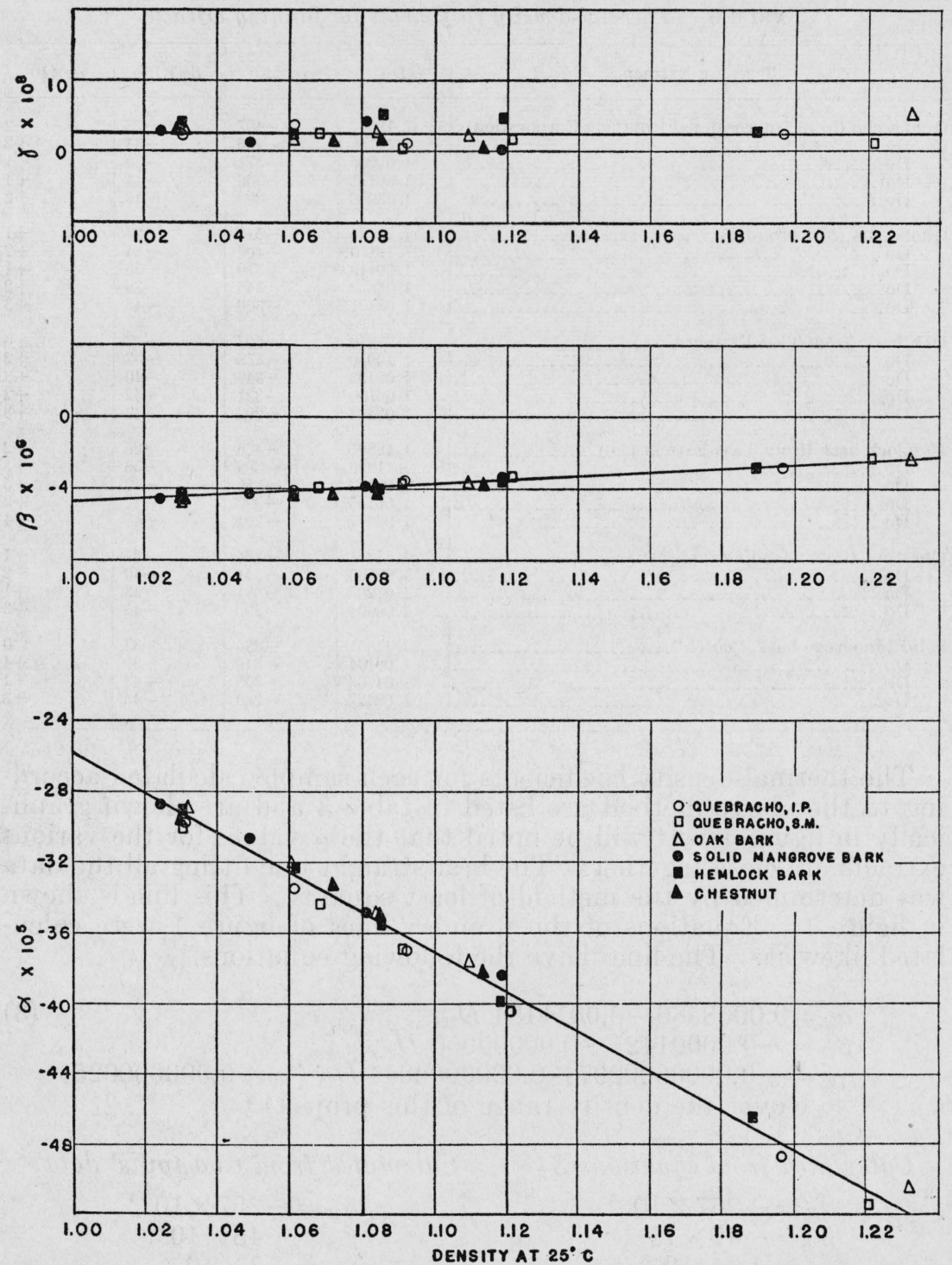

Figure 1.-Thermal-density coefficients $\alpha, \beta$, and $\gamma$ as functions of density. 


\section{TRANSFORMATION OF DATA TO $60^{\circ} \mathrm{F}$}

The coefficients given in the preceding section apply only at $25^{\circ} \mathrm{C}$. However, barkometer, Twaddle, and Baumé hydrometers (the hydrometers usually employed for measuring the densities of tanning extracts) are all standardized at $60^{\circ} \mathrm{F}$. Consequently, equation 2, representing the expansion of the tanning extracts at $25^{\circ} \mathrm{C}$, and the corresponding coefficients have been transformed in such a way that by their use the density at any temperature can be calculated from the density at $60^{\circ} \mathrm{F}\left(15.56^{\circ} \mathrm{C}\right)$. When so transformed the equation becomes

$$
D_{t}=D_{15.56}+\alpha_{15.56}(t-15.56)+\beta_{15.56}(t-15.56)^{2}+\gamma_{15.56}(t-15.56)^{3},
$$

in which

$$
\begin{aligned}
& \alpha_{15.56}=\alpha_{25}+2 \beta_{25}(15.56-25)+3 \gamma_{25}(15.56-25)^{2} \\
& \beta_{15.56}=\beta_{25}+3 \gamma_{25}(15.56-25) \\
& \gamma_{15.56}=\gamma_{25} \bullet
\end{aligned}
$$

The density at $15.56^{\circ}$ may be obtained from density at $25^{\circ} \mathrm{C}$ by use of equation 2.

Use of the transformed equation 4 is still dependent indirectly through the coefficients upon density at $25^{\circ} \mathrm{C}$. However, a table of relationships may be set up easily over the desired range between density at $25^{\circ} \mathrm{C}$; density at $15.56^{\circ} \mathrm{C}$ (computed from density at $25^{\circ} \mathrm{C}$ ); and $\alpha_{15.56}, \beta_{15.56}$, and $\gamma_{15.56}$ (computed from $\alpha_{25}, \beta_{25}$, and $\gamma_{25}$ ).

For calculation of the hydrometer correction tables the equation has been transformed still farther. Changed to a specific gravity basis, the equation becomes

$$
\begin{aligned}
& \text { Sp gr } \underset{15.56}{t}=\operatorname{Spgr}_{\frac{15.56}{15.56}}+\alpha_{\frac{15.56}{15.56}}(t-15.56) \\
& +\beta_{\frac{15.56}{15.56}}(t-15.56)^{2}+\gamma_{\frac{15.56}{15.56}}(t-15.56)^{3} .
\end{aligned}
$$

The coefficients $\alpha_{\frac{15.56}{15.56}}, \beta_{\frac{15.56}{15.56}}$, and $\gamma_{\frac{15.56}{15.56}}$ of this equation are equal to the corresponding coefficients $\alpha_{15.56}, \beta_{15.56}$, and $\gamma_{15.56}$ divided by the density of water at $15.56^{\circ} \mathrm{C}$. Table 4 provides numerical values for these coefficients. Hence, by the combined use of table 4 and equation 5, the specific gravity of a vegetable tanning extract at any temperature within the range may be calculated from its specific gravity at $60^{\circ} / 60^{\circ} \mathrm{F}$. 
TABLE 4.-Thermal density coefficients for tanning extracts for use with equation (5)

\begin{tabular}{|c|c|c|c|}
\hline $\begin{array}{l}\text { Specific gravity } \\
\text { at } \frac{15.56^{\circ}}{15.56^{\circ}} \mathrm{C}\left(\frac{60^{\circ}}{60^{\circ}} \mathrm{F}\right)\end{array}$ & $\frac{\alpha_{15.56}}{15.56} \times 10^{8}$ & $\frac{\beta_{15.56}}{15.56} \times 10^{7}$ & $\frac{\gamma_{15.56}}{15.56} \times 10^{8}$ \\
\hline $\begin{array}{l}1.000 \\
1.005 \\
1.010 \\
1.015 \\
1.020\end{array}$ & $\begin{array}{l}-160 \\
-167 \\
-173 \\
-180 \\
-186\end{array}$ & $\begin{array}{l}-54 \\
-54 \\
-54 \\
-53 \\
-53\end{array}$ & $\begin{array}{l}+3 \\
+3 \\
+3 \\
+3 \\
+3\end{array}$ \\
\hline $\begin{array}{l}1.025 \\
1.030 \\
1.035 \\
1.040 \\
1.045\end{array}$ & $\begin{array}{l}-192 \\
-199 \\
-205 \\
-212 \\
-218\end{array}$ & $\begin{array}{l}-52 \\
-52 \\
-51 \\
-51 \\
-50\end{array}$ & $\begin{array}{l}+3 \\
+3 \\
+3 \\
+3 \\
+3\end{array}$ \\
\hline $\begin{array}{l}\text { 1. } 050 \\
\text { 1. } 055 \\
\text { 1. } 060 \\
\text { 1. } 065 \\
\text { 1. } 070\end{array}$ & $\begin{array}{l}-224 \\
-231 \\
-237 \\
-244 \\
-250\end{array}$ & $\begin{array}{l}-50 \\
-49 \\
-49 \\
-48 \\
-48\end{array}$ & $\begin{array}{l}+3 \\
+3 \\
+3 \\
+3 \\
+3\end{array}$ \\
\hline $\begin{array}{l}\text { 1. } 075 \\
\text { 1. } 080 \\
\text { 1. } 085 \\
\text { 1. } 090 \\
\text { 1. } 095\end{array}$ & $\begin{array}{l}-256 \\
-263 \\
-269 \\
-275 \\
-282\end{array}$ & $\begin{array}{l}-48 \\
-47 \\
-47 \\
-46 \\
-46\end{array}$ & $\begin{array}{l}+3 \\
+3 \\
+3 \\
+3 \\
+3\end{array}$ \\
\hline $\begin{array}{l}\text { 1. } 100 \\
\text { 1. } 105 \\
\text { 1. } 110 \\
\text { 1. } 115 \\
\text { 1. } 120\end{array}$ & $\begin{array}{l}-288 \\
-294 \\
-301 \\
-308 \\
-314\end{array}$ & $\begin{array}{l}-45 \\
-45 \\
-44 \\
-44 \\
-43\end{array}$ & $\begin{array}{l}+3 \\
+3 \\
+3 \\
+3 \\
+3\end{array}$ \\
\hline
\end{tabular}

\section{CALCULATION OF HYDROMETER CORRECTIONS}

By use of equation 5 , specific gravities at $10^{\circ}, 20^{\circ}, 25^{\circ}, 30^{\circ}, 35^{\circ}$, and $40^{\circ} \mathrm{C}$ with reference temperature $15.56^{\circ} \mathrm{C}$ were calculated corresponding to each specific gravity at $15.56^{\circ} / 15.56^{\circ} \mathrm{C}$ at intervals of 0.005 throughout the desired range. These calculated specific gravities represent the readings on hydrometers which are correct, respectively, at the temperatures of the calculation. The corresponding readings on hydrometers standardized at $60^{\circ} / 60^{\circ} \mathrm{F}$ were found by applying the conversion of density basis formula for hydrometers. ${ }^{3}$

$$
\operatorname{Spgr}_{\frac{t}{15.56^{\circ} \mathrm{C}}}=\operatorname{Spgr}_{\frac{15.5{ }^{\circ} \mathrm{C}}{15.56^{\circ} \mathrm{C}}}+\Delta \operatorname{Sp}_{\frac{15.56^{\circ} \mathrm{C}}{15.56^{\circ} \mathrm{C}}}
$$

in which $\Delta=0.000023(15.56-t)$.

Interpolation was used to find the hydrometer readings at the temperature intervals corresponding to the same true specific gravity at $60^{\circ} / 60^{\circ} \mathrm{F}$. These values were plotted, one axis representing temperature and the other representing the hydrometer reading. A series of smooth curves was obtained consisting of a curve for each true specific gravity. From each curve were read the hydrometer readings for each degree change in temperature. The table was completed by interpolation to obtain columns for each 0.001 change in specific gravity. The table is given in abbreviated form with this paper. See table 5.

3 The hydrometers are assumed to be made of glass with a coefficient of cubical expansion equal to 0.000023 per degree centigrade. This figure in the equation may be replaced by the proper coefficients and tables calculated for hydrometers of other kinds of glass, or the tables presented here may be used with hydrometers of other glasses if a correction is applied to the reading of the hydrometer to obtain the corresponding reading on a hydrometer of glass with a coefficient equal to 0.000023 per degree centigrade. The correction is $+(\alpha-0.000023)(15.56-t) R$, in which $R$ is the reading of the hydrometer made at temperatures $t$, and $\alpha$ is the coefficient of the glass of which the hydrometer is made.

The largest error that is made by the use of the present table for Pyrex hydrometers without applying a correction is only 0.00036 in specific gravity $\left(0.4^{\circ} \mathrm{bk}\right)$. This error would occur at the maximal density and temperature. 
TABLE 5.-Hydrometer correction data

Reduction of observed specific gravities to specific gravities at $60^{\circ} / 60^{\circ} \mathrm{F}$.

\begin{tabular}{|c|c|c|c|c|c|c|c|}
\hline \multirow{3}{*}{$\begin{array}{c}\text { Observed } \\
\text { temperature }\end{array}$} & \multicolumn{7}{|c|}{ Observed specific gravity } \\
\hline & 1.0000 & 1.0200 & 1.0400 & 1.0600 & 1.0800 & 1.1000 & 1.1200 \\
\hline & \multicolumn{7}{|c|}{ Corresponding specific gravity at $60^{\circ} / 60^{\circ} \mathrm{F}$} \\
\hline $\begin{array}{l}50 \ldots \\
51 \ldots- \\
52 \ldots- \\
53 \ldots- \\
54 \ldots\end{array}$ & -- & $\begin{array}{l}1.0193 \\
1.0193 \\
1.0194 \\
1.0194 \\
1.0195\end{array}$ & $\begin{array}{l}1.0391 \\
1.0392 \\
1.0393 \\
1.0394 \\
1.0395\end{array}$ & $\begin{array}{l}\text { 1. } 0590 \\
\text { 1. } 0591 \\
\text { 1. } 0592 \\
\text { 1. } 0593 \\
\text { 1. } 0594\end{array}$ & $\begin{array}{l}1.0788 \\
1.0790 \\
1.0791 \\
1.0792 \\
1.0793\end{array}$ & $\begin{array}{l}1.0987 \\
1.0988 \\
1.0989 \\
1.0991 \\
1.0992\end{array}$ & $\begin{array}{l}1.1186 \\
1.1187 \\
1.1188 \\
1.1190 \\
1.1191\end{array}$ \\
\hline $\begin{array}{l}55 \ldots \\
56 \ldots \\
57 . . \\
58 \ldots \\
59 \ldots\end{array}$ & (n) & $\begin{array}{l}1.0196 \\
1.0197 \\
1.0197 \\
1.0198 \\
1.0199\end{array}$ & $\begin{array}{l}1.0395 \\
1.0396 \\
1.0397 \\
1.0398 \\
1.0399\end{array}$ & $\begin{array}{l}\text { 1. } 0595 \\
\text { 1. } 0596 \\
\text { 1. } 0597 \\
\text { 1. } 0598 \\
\text { 1. } 0599\end{array}$ & $\begin{array}{l}1.0794 \\
1.0796 \\
1.0797 \\
1.0798 \\
1.0799\end{array}$ & $\begin{array}{l}\text { 1. } 0993 \\
1.0995 \\
\text { 1. } 0996 \\
\text { 1. } 0997 \\
1.0998\end{array}$ & $\begin{array}{l}1.1192 \\
1.1194 \\
1.1196 \\
1.1197 \\
1.1198\end{array}$ \\
\hline $\begin{array}{l}60 \ldots \\
61 \ldots \\
62 . . \\
63 \ldots \\
64 \ldots\end{array}$ & $\begin{array}{l}1.0000 \\
1.0001 \\
1.0001 \\
1.0002 \\
1.0003\end{array}$ & $\begin{array}{l}1.0200 \\
1.0201 \\
1.0202 \\
1.0203 \\
1.0204\end{array}$ & $\begin{array}{l}1.0400 \\
1.0401 \\
1.0402 \\
1.0403 \\
1.0404\end{array}$ & $\begin{array}{l}\text { 1. } 0600 \\
\text { 1. } 0601 \\
\text { 1. } 0602 \\
\text { 1. } 0604 \\
\text { 1. } 0605\end{array}$ & $\begin{array}{l}1.0800 \\
1.0801 \\
1.0803 \\
1.0804 \\
1.0806\end{array}$ & $\begin{array}{l}1.1000 \\
1.1001 \\
1.1003 \\
1.1004 \\
1.1005\end{array}$ & $\begin{array}{l}1.1200 \\
1.1202 \\
1.1204 \\
1.1205 \\
1.1207\end{array}$ \\
\hline $\begin{array}{l}65 \ldots \\
66 \ldots- \\
67 \ldots- \\
68 \ldots \\
69-.\end{array}$ & $\begin{array}{l}1.0004 \\
1.0005 \\
1.0006 \\
1.0007 \\
1.0008\end{array}$ & $\begin{array}{l}1.0205 \\
1.0206 \\
1.0207 \\
1.0208 \\
1.0210\end{array}$ & $\begin{array}{l}1.0406 \\
1.0407 \\
1.0408 \\
1.0409 \\
1.0411\end{array}$ & $\begin{array}{l}\text { 1. } 0606 \\
\text { 1. } 0608 \\
\text { 1. } 0609 \\
\text { 1. } 0610 \\
\text { 1. } 0612\end{array}$ & $\begin{array}{l}1.0807 \\
1.0808 \\
1.0810 \\
1.0812 \\
1.0813\end{array}$ & $\begin{array}{l}1.1008 \\
1.1009 \\
1.1011 \\
1.1013 \\
1.1014\end{array}$ & $\begin{array}{l}\text { 1. } 1208 \\
1.1210 \\
1.1212 \\
1.1214 \\
1.1216\end{array}$ \\
\hline $\begin{array}{l}70 \ldots . . \\
71-\ldots \\
72-. . \\
73-. . \\
74 \ldots\end{array}$ & $\begin{array}{l}1.0010 \\
1.0011 \\
1.0012 \\
1.0013 \\
1.0014\end{array}$ & $\begin{array}{l}1.0211 \\
1.0212 \\
1.0213 \\
1.0215 \\
1.0216\end{array}$ & $\begin{array}{l}\text { 1. } 0412 \\
\text { 1. } 0413 \\
1.0415 \\
1.0416 \\
1.0418\end{array}$ & $\begin{array}{l}\text { 1. } 0613 \\
\text { 1. } 0615 \\
\text { 1. } 0616 \\
\text { 1. } 0618 \\
\text { 1. } 0620\end{array}$ & $\begin{array}{l}1.0815 \\
1.0817 \\
1.0818 \\
1.0820 \\
1.0822\end{array}$ & $\begin{array}{l}1.1016 \\
1.1018 \\
1.1020 \\
1.1022 \\
1.1024\end{array}$ & $\begin{array}{l}\text { 1. } 1218 \\
1.1220 \\
1.1221 \\
1.1223 \\
1.1225\end{array}$ \\
\hline $\begin{array}{l}75 \ldots . . \\
76 \ldots-. \\
77-\ldots \\
78 \ldots \\
79 . . .\end{array}$ & $\begin{array}{l}1.0015 \\
1.0017 \\
1.0018 \\
1.0019 \\
1.0021\end{array}$ & $\begin{array}{l}1.0217 \\
1.0219 \\
1.0220 \\
1.0222 \\
1.0223\end{array}$ & $\begin{array}{l}1.0419 \\
1.0421 \\
1.0422 \\
1.0424 \\
1.0426\end{array}$ & $\begin{array}{l}\text { 1. } 0621 \\
\text { 1. } 0623 \\
\text { 1. } 0625 \\
\text { 1. } 0626 \\
\text { 1. } 0628\end{array}$ & $\begin{array}{l}1.0823 \\
1.0825 \\
1.0827 \\
1.0829 \\
1.0830\end{array}$ & $\begin{array}{l}1.1026 \\
1.1027 \\
1.1029 \\
1.1031 \\
1.1033\end{array}$ & $\begin{array}{l}1.1227 \\
1.1229 \\
1.1231 \\
1.1233 \\
1.1236\end{array}$ \\
\hline $\begin{array}{l}80 \ldots . . \\
81 \ldots . . \\
82 \ldots \\
83 \ldots . . \\
84 \ldots\end{array}$ & $\begin{array}{l}1.0022 \\
1.0024 \\
1.0025 \\
1.0027 \\
1.0028\end{array}$ & $\begin{array}{l}1.0225 \\
1.0226 \\
1.0228 \\
1.0230 \\
1.0231\end{array}$ & $\begin{array}{l}1.0427 \\
1.0429 \\
1.0430 \\
1.0432 \\
1.0434\end{array}$ & $\begin{array}{l}\text { 1. } 0630 \\
\text { 1. } 0632 \\
\text { 1. } 0634 \\
\text { 1. } 0636 \\
\text { 1. } 0638\end{array}$ & $\begin{array}{l}1.0832 \\
1.0834 \\
1.0836 \\
1.0838 \\
1.0840\end{array}$ & $\begin{array}{l}1.1035 \\
1.1037 \\
1.1039 \\
1.1041 \\
1.1043\end{array}$ & $\begin{array}{l}1.1238 \\
1.1240 \\
1.1242 \\
1.1244 \\
1.1246\end{array}$ \\
\hline $\begin{array}{l}85-. \\
86-. \\
87-- \\
88 .- \\
89 \ldots\end{array}$ & $\begin{array}{l}1.0030 \\
1.0031 \\
1.0033 \\
1.0035 \\
1.0036\end{array}$ & $\begin{array}{l}1.0233 \\
1.0234 \\
1.0236 \\
1.0238 \\
1.0240\end{array}$ & $\begin{array}{l}1.0436 \\
1.0438 \\
1.0439 \\
1.0441 \\
1.0443\end{array}$ & $\begin{array}{l}\text { 1. } 0639 \\
\text { 1. } 0641 \\
\text { 1. } 0643 \\
\text { 1. } 0645 \\
\text { 1. } 0647\end{array}$ & $\begin{array}{l}1.0842 \\
1.0844 \\
1.0846 \\
1.0848 \\
1.0850\end{array}$ & $\begin{array}{l}\text { 1. } 1046 \\
1.1048 \\
1.1050 \\
1.1052 \\
1.1054\end{array}$ & $\begin{array}{l}1.1248 \\
1.1251 \\
1.1253 \\
1.1256 \\
1.1258\end{array}$ \\
\hline $\begin{array}{l}90 \ldots \\
91-. . \\
92 \ldots- \\
93 .- \\
94 \ldots\end{array}$ & $\begin{array}{l}1.0038 \\
1.0040 \\
1.0041 \\
1.0043 \\
1.0045\end{array}$ & $\begin{array}{l}\text { 1. } 0241 \\
1.0243 \\
1.0245 \\
1.0247 \\
1.0249\end{array}$ & $\begin{array}{l}1.0445 \\
1.0447 \\
1.0449 \\
1.0451 \\
1.0453\end{array}$ & $\begin{array}{l}\text { 1. } 0649 \\
\text { 1. } 0651 \\
\text { 1. } 0653 \\
\text { 1. } 0655 \\
\text { 1. } 0657\end{array}$ & $\begin{array}{l}1.0852 \\
1.0855 \\
1.0857 \\
1.0859 \\
1.0861\end{array}$ & $\begin{array}{l}1.1057 \\
1.1059 \\
1.1062 \\
1.1064 \\
1.1066\end{array}$ & $\begin{array}{l}1.1260 \\
1.1262 \\
1.1265 \\
1.1267 \\
1.1270\end{array}$ \\
\hline $\begin{array}{l}95 \ldots \\
96 \ldots \\
97-\ldots \\
98 \\
99 \\
99 . .\end{array}$ & $\begin{array}{l}1.0047 \\
1.0048 \\
1.0050 \\
1.0052 \\
1.0054\end{array}$ & $\begin{array}{l}1.0251 \\
1.0253 \\
1.0255 \\
1.0257 \\
1.0259\end{array}$ & $\begin{array}{l}1.0455 \\
1.0457 \\
1.0459 \\
1.0461 \\
1.0464\end{array}$ & $\begin{array}{l}\text { 1. } 0659 \\
\text { 1. } 0662 \\
\text { 1. } 0664 \\
\text { 1. } 0666 \\
\text { 1. } 0668\end{array}$ & $\begin{array}{l}1.0864 \\
1.0866 \\
1.0868 \\
1.0870 \\
2.0872\end{array}$ & $\begin{array}{l}1.1068 \\
1.1070 \\
1.1073 \\
1.1075 \\
1.1078\end{array}$ & $\begin{array}{l}1.1272 \\
1.1274 \\
1.1277 \\
1.1280 \\
1.1282\end{array}$ \\
\hline 100 & 1.0056 & 1.0261 & 1.0466 & 1. 0671 & 1.0875 & 1. 1080 & 1.1285 \\
\hline
\end{tabular}


Barkometer, Twaddle, and Baumé tables were computed from the specific-gravity table. These tables are not included with this paper but will be published separately in Bureau Circular C449. The scope of the tables is as follows:

\begin{tabular}{|c|c|c|}
\hline Table & Range & Interval \\
\hline $\begin{array}{l}\text { Barkometer-1. } \\
\text { Baumé } \\
\text { Twaddle_-_- }\end{array}$ & $\begin{array}{l}0^{\circ} \text { to } 119^{\circ} \mathrm{bk} \\
\mathrm{O}^{\circ} \text { to } 15^{\circ} \mathrm{Be} \\
\mathrm{O}^{\circ} \text { to } 24^{\circ} \mathrm{TW}\end{array}$ & $\begin{array}{l}1.0 \\
1.0 \\
1.0\end{array}$ \\
\hline
\end{tabular}

True values corresponding to the above observed values are given to the nearest 0.1 degree for each degree change in temperature from $50^{\circ}$ to $100^{\circ} \mathrm{F}$. The relationship between the scales is

$$
\begin{aligned}
\text { Sp gr } 60^{\circ} / 60^{\circ} \mathrm{F} & =\frac{1000+^{\circ} \mathrm{bk}}{1000} \\
\text { Degrees barkometer } & =\frac{\operatorname{Spgr} 60^{\circ} / 60^{\circ} \mathrm{F}-1}{0.001} \\
\text { Sp gr } 60^{\circ} / 60^{\circ} \mathrm{F} & =\frac{1000+\left(^{\circ} \mathrm{Tw} \times 5\right)}{1000} \\
\text { Degrees Twaddle } & =\frac{\operatorname{Spgr~} 60^{\circ} / 60^{\circ} \mathrm{F}-1}{0.005} \\
\text { Sp gr } 60^{\circ} / 60^{\circ} \mathrm{F} & =\frac{145}{145-{ }^{\circ} \mathrm{Bé}} \\
\text { Degrees Baumé } & =145-\frac{145}{\operatorname{Spgr~} 60^{\circ} / 60^{\circ} \mathrm{F}}
\end{aligned}
$$

\section{APPLICABILITY AND ACCURACY OF THE COEFFI- CIENTS AND DERIVED CORRECTION TABLES}

The thermal-density coefficients of equations 3 and table 4 were computed from the observed densities of several representative tanning extracts. Duplicate observations agree, in general, within three units in the fifth decimal place of the density value. These observed densities were subjected to an adjustment by the method of least squares to determine the most probable density at each temperature. Hence, errors in the original observation and their reduction are small in comparison with the error due to assuming one value for all extracts. The accuracy of the specific gravity of any particular extract computed from the coefficients is dependent upon the closeness with which the rate of expansion of that particular extract agrees with the composite rate.

Conclusions as to the degree of accuracy obtainable when the composite coefficients of equations 3 are used may be drawn from an examination of the graphs in figure 1 . Slight variations between coefficients of the different types of extracts studied are evident, while the agreement between different brands of the same type is remarkable. For example, the points representing the $\alpha$ coefficients of two brands 
of quebracho vary little from a straight line, but this line is definitely below the least squares line. Further examination shows the coefficient farthest from the least squares line to be that of quebracho (SM brand) with density $1.12 \mathrm{~g} / \mathrm{ml}$. The observed value of $\alpha$ at this point is $-407 \times 10^{-6}$. The least squares value is $-396 \times 10^{-6}$. The difference is 0.000011 . This differenceintroduces an error of 0.00016 in the density calculated at $40^{\circ} \mathrm{C}$, the temperature farthest from the standard temperature $25^{\circ} \mathrm{C}$. When the $\beta$ and $\gamma$ values are considered, the difference caused by using the least squares value is 0.00015 . Over most of the temperature range and for most of the extracts the discrepancy is much less.

Since the hydrometer correction tables are based on the data discussed in the preceding paragraph, they should be accurate to the same degree if calculated with like sensitivity. Calculation of corrections for the barkometer table was made to $0.1^{\circ} \mathrm{bk}$. This hydrometer is usually graduated in degrees barkometer, equivalent to $0.001 \mathrm{sp}$ gr at $60^{\circ} / 60^{\circ} \mathrm{F}$. Probable accuracy of the correction table, then, is much greater than that with which the hydrometer is likely to be read.

The barkometer table had been compared with the table ${ }^{4}$ supplied by The American Leather Chemists' Association as the basis that is being used in the leather industry. A greater accuracy as well as a greater range has been attempted in the new table. The two agree exactly at low densities and temperatures but diverge as either temperature or density increases. This difference amounts to $1.1^{\circ} \mathrm{bk}$ for a reading of $60^{\circ}$ bk at $100^{\circ} \mathrm{F}$, the larger value being given by the new table.

\section{CONCLUSION}

The thermal-density coefficients have been presented in such a manner that they may be used for calculation of the density of a tanning extract at any other temperature within the range covered by the investigation if the density at either $25^{\circ} \mathrm{C}$ or $60^{\circ} \mathrm{F}$ is known.

Data for correcting hydrometer readings, calculated with the aid of these coefficients, have been prepared in tabular form at the request of the tanning industry to meet the needs of that industry. It is believed that they are applicable to all vegetable tanning extracts included in this investigation without the introduction of errors in excess of those that unavoidably occur in the reading of hydrometers under industrial laboratory conditions.

Washington, June 27, 1944.

${ }^{4}$ This table may be found in J. A. Wilson, Modern Practice in Leather Manufacture, p. 287 (Reinhold Publishing Corporation, New York, N. Y., 1941). 